Corrigendum: The Green's function for the radiative transport equation in the slab geometry

This article has been downloaded from IOPscience. Please scroll down to see the full text article.

2012 J. Phys. A: Math. Theor. 45459501

(http://iopscience.iop.org/1751-8121/45/45/459501)

View the table of contents for this issue, or go to the journal homepage for more

Download details:

IP Address: 141.211.173.82

The article was downloaded on 25/06/2013 at 20:31

Please note that terms and conditions apply. 


\title{
Corrigendum: The Green's function for the radiative transport equation in the slab geometry
}

2010 J. Phys. A: Math. Theor. 43065402

\author{
Manabu Machida $^{1}$, George Y Panasyuk ${ }^{2}$, John C Schotland ${ }^{1}$ \\ and Vadim A Markel $^{3}$ \\ ${ }^{1}$ Department of Mathematics, University of Michigan, Ann Arbor, MI 48109, USA \\ 2 Aerospace Systems Directorate, Air Force Research Laboratory, Wright-Patterson Air Force \\ Base, OH 45433, USA \\ ${ }^{3}$ Departments of Radiology and Bioengineering and the Graduate Group in Applied Mathematics \\ and Computational Science, University of Pennsylvania, Philadelphia, PA 19104, USA \\ E-mail:mmachida@umich.edu, George.Panasyuk.ctr@wpafb.af.mil, schotland@umich.edu and \\ vmarkel@mail.med.upenn.edu
}

Received 1 October 2012

Published 23 October 2012

Online at stacks.iop.org/JPhysA/45/459501

In the previously published paper [1], we have computed the energy density of electromagnetic radiation $u(\mathbf{r})$ by the analytical method developed in the paper (the method of rotated reference frames (MRRF)) and by the Monte Carlo method. The results were compared in figure 9 of [1]. The figure displays a discrepancy between the two curves that we attributed to unknown imprecisions inherent in both methods. However, we have discovered an error in the computer code which was used to produce the MRRF curve. When this error was corrected, much better agreement was obtained. The corrected figure is presented below. We thus conclude that,

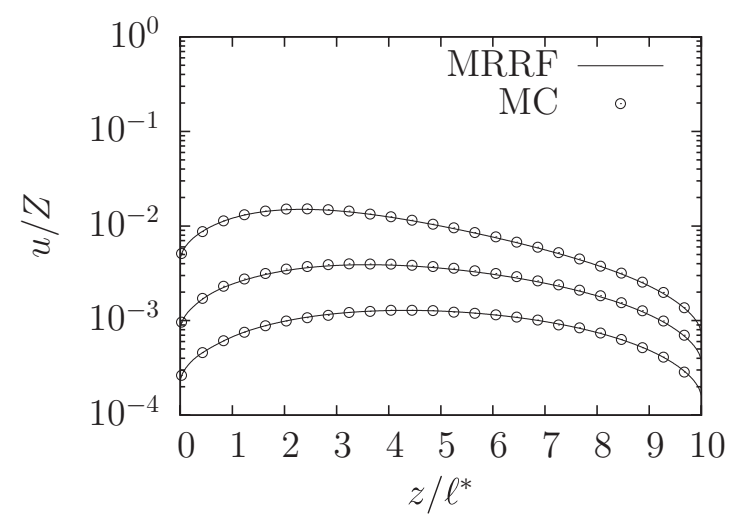

Figure 9. Density $u(z, \rho)$ computed by the MRRF and by Monte Carlo simulations for a slab with $L=10 \ell^{*}$. From top to bottom, the curves correspond to $\rho=4 \ell^{*}, \rho=7 \ell^{*}$ and $\rho=10 \ell^{*}$. Normalization factor $Z=I_{0} /\left(\ell^{*}\right)^{2}$. 
for the parameters considered, the MRRF agrees with Monte Carlo simulations with good precision. This finding is consistent with the recent paper of Liemert and Kienle [2].

We note that the other figures or simulation data in [1] have not been affected by the error mentioned above.

\section{Acknowledgments}

This research was supported by the NSF under grants EEC-0615857 and DMS-0554100.

\section{References}

[1] Machida M, Panasyuk G Y, Schotland J C and Markel V A 2010 The Green's function for the radiative transport equation in the slab geometry J. Phys. A: Math. Theor. 43065402

[2] Liemert A and Kienle A 2012 Light transport in three-dimensional semi-infinite scattering media J. Opt. Soc. Am. A 29 1475-81 